\title{
MSK1 downregulation is associated with neuronal and astrocytic apoptosis following subarachnoid hemorrhage in rats
}

\author{
BO NING ${ }^{1,2^{*}}$, GENG GUO ${ }^{2,3^{*}}$, HONG LIU $^{4}$, LEI NING ${ }^{5}$, BAO-LIANG SUN ${ }^{6}$, \\ ZHEN LI ${ }^{7}$, SHUO WANG ${ }^{2}$, ZHENG-WEN LV ${ }^{7}$ and CUN-DONG FAN ${ }^{6}$ \\ ${ }^{1}$ Department of Neurosurgery, Guangzhou Red Cross Hospital, Jinan University, Guangzhou, Guangdong 510220; \\ ${ }^{2}$ Department of Neurosurgery, Beijing Tiantan Hospital, Capital Medical University, Beijing 100050; \\ ${ }^{3}$ Department of Neurosurgery, The First Hospital, Shanxi Medical University, Taiyuan, Shanxi 030001; \\ ${ }^{4}$ Department of Scientific Research, Taian Central Hospital; ${ }^{5}$ Department of Medical Records, \\ Affiliated Hospital of Taishan Medical University; ${ }^{6} \mathrm{Key} \mathrm{Lab}$ of Cerebral Microcirculation in Universities of Shandong, \\ Taishan Medical University; ${ }^{7}$ Department of Neurosurgery, Taian Central Hospital, Taian, Shandong 271000, P.R. China
}

Received October 21, 2015; Accepted April 28, 2017

DOI: $10.3892 / 01.2017 .6496$

\begin{abstract}
MSK (mitogen- and stress-activated protein kinase) proteins are a family of mitogen-activated protein kinases. MSKs represent a novel type of pro-survival genes, potentially enhancing the phosphorylation of $\mathrm{Bcl} 2$-associated agonist of cell death. However, MSK's function and expression are poorly understood in the central nervous system. In the present study, a subarachnoid hemorrhage (SAH) model was established in SD rats and the expression of MSK1 in the brain subsequent to experimental SAH was investigated. In response to $\mathrm{SAH}$, MSK1 mRNA and protein levels gradually declined, reaching the lowest point at 3 days, and increased thereafter. The expression of active caspase-3 was negatively correlated with MSK1 level. Colocalization and correlating changes in expression of MSK1 and active caspase- 3 at neurons and astrocytes indicated that MSK1 downregulation may contribute to SAH-induced apoptosis, validating that MSK1 may be involved in the pathophysiology of the brain cortex subsequent to SAH.
\end{abstract}

Correspondence to: Professor Shuo Wang, Department of Neurosurgery, Beijing Tiantan Hospital, Capital Medical University, No. 6 Tiantan Xili, Chongwen, Beijing 100050, P.R. China E-mail: captain9858@vip.sina.com

Dr Zheng-Wen Lv, Department of Neurosurgery, Taian Central Hospital, No. 29 Long Tan Road, Taian, Shandong 271000, P.R. China

E-mail: lvzhengwen1388@163.com

*Contributed equally

Key words: subarachnoid hemorrhage, mitogen- and stress-activated protein kinase 1 , neuronal nuclear antigen, glial fibrillary acidic protein, apoptosis

\section{Introduction}

Subarachnoid hemorrhage (SAH), typically caused by the rupture of an intracranial aneurysm, is a fatal disease with high associated morbidity and mortality rates (1). The annual incidence of SAH is $\sim 22.5$ cases per 100,000 population, according to a World Health Organization study (2). SAH has a mortality rate of $40-50 \%$, and the prognosis of survivors is poor $(3,4)$. This is associated with a notable economic burden as $50 \%$ of SAH patients are $<55$ years old (5-7). Experimental evidence supports the hypothesis that neurological deterioration and a poor outcome are a response to cerebral vasospasm subsequent to SAH (8). However, a number of patients undergo neurological deterioation without an accompanying vasospasm and preventing vasospasm may not always improve the clinical outcome (9). There is research to suggest that delayed neuronal and astrocytic apoposis is an important contributor to a poor outcome in patients with SAH $(10,11)$. The present study aimed to assess the factors responsible for this process and outline the associated future possibilities for the improvement of SAH treatment.

MSK (mitogen- and stress-activated protein kinase) proteins are a particularly interesting family of mitogen-activated protein kinases. They were originally identified through their homology with the N-terminal ribosomal S6 kinase domain (12). MSK1 is a nuclear protein kinase with two kinase domains, including a C-terminal kinase domain related to the $\mathrm{Ca}^{2+} / \mathrm{calmodulin}$-dependent protein kinase family and an $\mathrm{N}$-terminal kinase domain related to the AGC kinase family $(13,14)$. It can be activated downstream of the mitogen-activated protein kinase (MAPK) 2/1 or MAPK 11/14 cascades with the phosphorylation of Thr581 in the C-terminal kinase domain (15). Once activated, the $\mathrm{N}$-terminal domain phosphorylates a variety of substrates, including nuclear factor $\kappa \mathrm{B}(\mathrm{NF} \kappa \mathrm{B})$, cAMP responsive element binding protein (CREB) (16), histone subunit H3, and high-mobility group nucleosome binding domain 1 (HMGN1). The major role of MSKs in the CNS is to regulate the immediate early (IE) 
genes, plasticate neuronal synapses and promote cytokine production. The number of identified MSK1 targets continues to increase (17). It was previously established that MSKs are a novel type of pro-survival gene that enhances the phosphorylation of Bcl-2-associated death promoter (Bad) (18). Phosphorylation of Bad at Ser112 in response to growth factors or cytokines is a common mechanism for the promotion of cell survival; MSK1 knockdown was previously demonstrated to suppress Bad phosphorylation after calcium ionophore A23187 treatment in neuronal cells (18).

However, the function and expression of MSK1 in the central nervous system (CNS) have yet to be well-characterized. It is established that MSK1 is highly expressed in the nervous system (19), but its function is not well understood. In the present study, the expression and distribution of MSK1 in the brain were examined following the experimental induction of SAH in rats. The study aimed to identify the physiological functions of MSK1 and the molecular mechanisms underlying lesion and repair in the CNS.

\section{Materials and methods}

Animals and surgical procedures. A total of 48 3-months old male Sprague-Dawley rats (280-320 g) were used. Rats were kept in the laboratory's temperature of $20^{\circ} \mathrm{C}$ and the humidity was $60 \%$. The rats were raised with a 12 -h light/dark cycle and free access to water and food. The animals were anesthetized with $10 \%$ chloral hydrate and positioned in a stereotactic frame with their heads tilted $\sim 30^{\circ}$ downwards. A midline scalp incision was made in the neck and the atlanto-occipital membrane was exposed subsequent to separating the muscle layers. The atlanto-occipital membrane was punctured with a needle (Fig. 1), and $0.3 \mathrm{ml}$ autologous arterial blood was injected into the cisterna magna with a squirt pump within $10 \mathrm{~min}$. A second injection of blood was performed with the same method following a days recovery. The control group were injected with $0.3 \mathrm{ml}$ sterile saline. Rats had free access to water and food during recovery from anesthesia. The animals with induced SAH were randomly divided into five sub-groups and sacrificed by decapitation on day $1,3,5,7$ or 14 post-SAH, ( $n=6$; Fig. 1). A further 6 rats with SAH were sacrificed with ventricle perfusion for immunohistochemical and immunofluorescence studies on day 3. An additional control group, sham animals $(n=6)$, experienced the same surgery process without the injection into the cisterna magna. Sham group animals were then sacrificed $24 \mathrm{~h}$ after the sham operation. All rats were supplied by Taishan Medical University (Taian, China) and all surgical interventions and postoperative animal care were performed in accordance with the Guide for the Care and Use of Laboratory Animals (National Research Council, 1996, USA) and were approved by the Ethics Committee of Animal Experiments of Taishan Medical University. All surgery was performed under $10 \%$ chloral hydrate and every effort was made to minimize suffering.

Western blotting. For western blot analysis, brain tissues were homogenized in lysis buffer ( $1 \%$ sodium deoxycholate, $50 \mathrm{mmol} / 1$ Tris, $1 \% \mathrm{NP}-40,1 \%$ Triton X-100, $5 \mathrm{mmol} / 1$ EDTA, $1 \%$ SDS, $1 \mathrm{mmol} / 1$ phenylmethane sulfonyl fluoride, $10 \mu \mathrm{g} / \mathrm{ml}$ aprotinin and $1 \mu \mathrm{g} / \mathrm{ml}$ leupeptin) and clarified by centrifuging at $14,000 \times \mathrm{g}$ for $15 \mathrm{~min}$ at $4^{\circ} \mathrm{C}$, from which the supernatant was collected. Then a BCA kit (Beyotime Institute of Biotechnology, Haimen, China) was used to determinate the protein concentration. Samples (80 $\mu \mathrm{g} /$ lane) were subjected to $10 \%$ SDS-PAGE for $40 \mathrm{~min}$ at $70 \mathrm{~V}$ followed by $90 \mathrm{~min}$ at $120 \mathrm{~V}$ and then transferred onto a PVDF membrane by a transfer apparatus at $180 \mathrm{~mA}$ for $2.5 \mathrm{~h}$. The membrane was blocked with $5 \%$ skimmed milk for $2 \mathrm{~h}$ at $20^{\circ} \mathrm{C}$ and then incubated with the appropriate primary antibodies (MSK1, ab81294, 1:200, Abcam, Cambridge, UK; active caspase-3, ab49822, 1:200, Abcam; neuronal nuclear antigen, ab177487, 1:100, Abcam; glial fibrillary acidic protein, sc-71143, 1:100, Santa Cruz Biotechnology, Inc., Dallas, TX, USA) at $4^{\circ} \mathrm{C}$ overnight. The membrane was washed three times in TBST and incubated with goat-anti-rabbit (sc-2030, 1:500, Santa Cruz Biotechnology, USA) or goat-anti-mouse IgG (sc-2031, 1:500, Santa Cruz Biotechnology, USA) conjugated to horseradish peroxidase antibody for $2 \mathrm{~h}$ at room temperature. The blotted protein bands were developed with enhanced chemiluminescence reagent (Thermo Fisher Scientific, Inc.) exposed on X-ray film. The relative density of each band compared with GADPH was estimated with Scion Image software version 4.0.3.2 (Scion Corporation, Frederick, MD, USA).

Double immunofluorescence staining. Brain tissue was fixed with $4 \%$ paraformaldehyde for $3 \mathrm{~h}$, then $20 \%$ saccharose solution for 2 days, then $30 \%$ sucrose solution for 2 days to remove water from the sample, all these procedures were performed at $4^{\circ} \mathrm{C}$. Sections of $8 \mu \mathrm{m}$ thickness were prepared and blocked with $5 \%$ normal fetal bovine serumin in PBS containing $0.1 \%$ Triton $\mathrm{X}-100$ for $2 \mathrm{~h}$. The slices were then incubated overnight at $4^{\circ} \mathrm{C}$ with primary antibodies against MSK1 (cat. no., ab81294, 1:200, Abcam); active caspase-3 (cat. no., ab49822, 1:200); neuronal nuclear antigen (cat. no., ab177487, 1:100, Abcam); glial fibrillary acidic protein (cat. no., sc-71,143, 1:100, Santa Cruz Biotechnology, Inc., Dallas, TX, USA). Subsequently, secondary antibodies goat-anti-rabbit IgG (cat. no., sc-2030, 1:500, Santa Cruz Biotechnology, Inc.) were added and incubated for $2 \mathrm{~h}$ at room temperature in the dark. Following 3 washes in PBS, the slides were observed under a fluorescence microscope (Leica Microsystems GmbH, Wetzlar, Germany). Negative controls omitted the primary antibodies.

Immunohistochemistry. Frozen cross-sections $(8 \mu \mathrm{m})$ were prepared and blocked with $5 \%$ fetal bovine serum (Gibco; Thermo Fisher Scientific, Inc.) in PBS for $2 \mathrm{~h}$ at room temperature. Then each of the sections was incubated with the anti-MSK1 antibody (cat. no., ab81294, 1:200, Abcam) overnight at $4^{\circ} \mathrm{C}$ followed by incubation in biotinylated Goat Anti-Rat IgG Antibody (cat. no., BP-9400, 1:500, Vector Laboratories, Inc., Burlingame, CA, USA). Staining was visualized with 3,3'-diaminobenzidine (DAB, Vector Laboratories, Inc.). Staining was visualized with 3,3'-diaminobenzidine. Cells with strong or moderate brown staining were considered as positive.

Reverse transcription-quantitative polymerase chain reaction (RT-qPCR). TRIzol reagent (Takara Biotechnology Co., Ltd., Dalian, China) was used to isolate RNA from rat tissues and the concentration of RNA was measured with spectrophotometric 

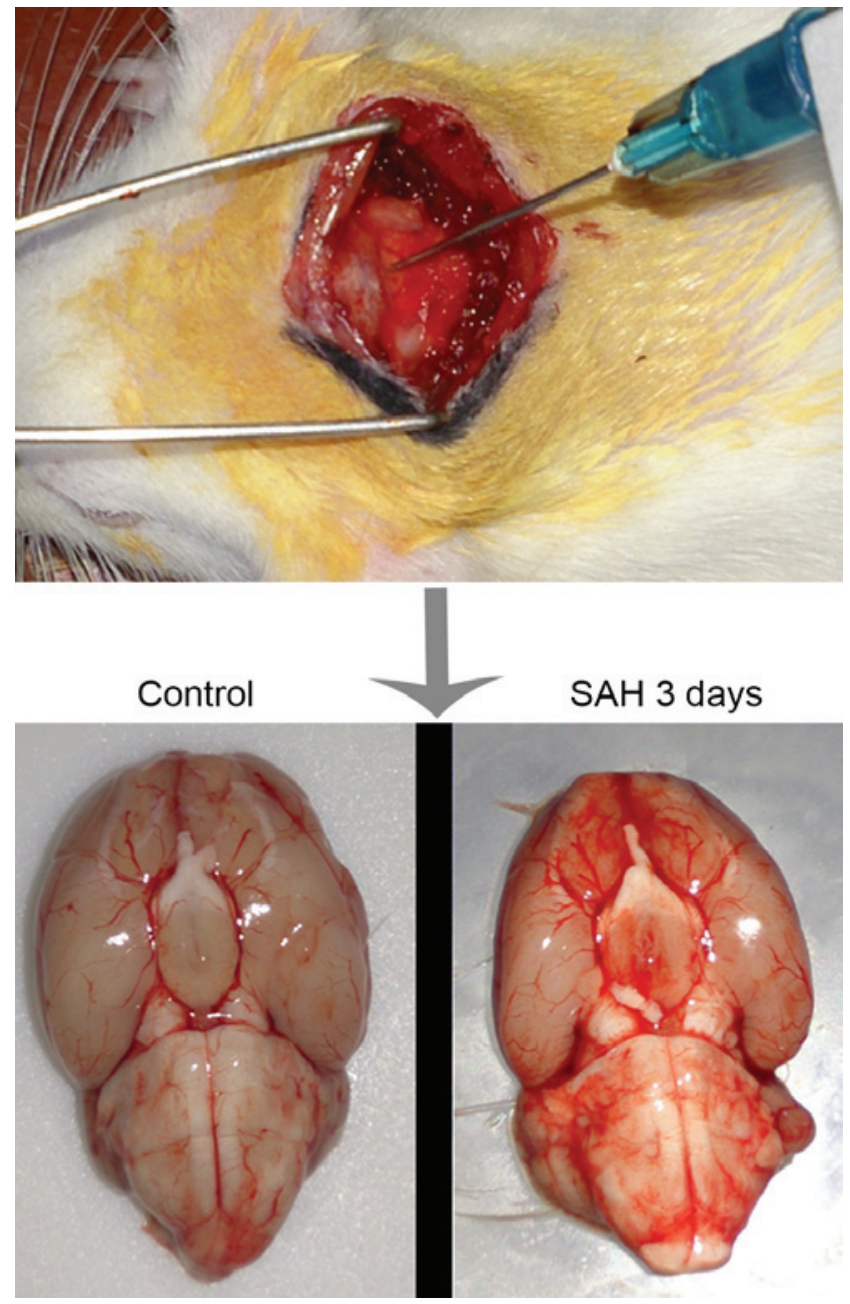

Figure 1. Autologous arterial blood was injected into the cisterna magna to simulate SAH. Representative images of a control rat brain and a rat brain harvested 3 days subsequent to SAH are included. SAH, subarachnoid hemorrhage.

analysis (optical density at 260/280). Total RNA was reversely transcribed into cDNA using a 25- $\mu 1$ mixture [Primer Mix (12 $\mu \mathrm{l})$, 5xRT Reaction Buffer (5 $\mu \mathrm{l}), 25 \mathrm{mM}$ dNTPs $(1 \mu \mathrm{l})$, $25 \mathrm{U} / \mu 1$ RNase Inhibitor $(1 \mu \mathrm{l}), 200 \mathrm{U} / \mu \mathrm{l}$ M-MLV Rtase $(1 \mu \mathrm{l})$, Oligo (dt) $18(1 \mu \mathrm{l})$ and $\mathrm{ddH}_{2} \mathrm{O}$ (DNase-free; $\left.\left.4 \mu \mathrm{l}\right)\right]$ at $37^{\circ} \mathrm{C}$ for $60 \mathrm{~min}, 85^{\circ} \mathrm{C}$ for $5 \mathrm{~min}$ and $4^{\circ} \mathrm{C}$ for $5 \mathrm{~min}$. cDNA was used as template for PCR (Prism 7300 Real-Time PCR System, Applied Biosystems; Thermo Fisher Scientific, Inc., USA) and the mixture used for PCR included SYBR-Green Mix (12.5 $\mu$; Invitrogen; Thermo Fisher Scientific, Inc.), forward primer $(0.5 \mu \mathrm{l})$, reverse primer $(0.5 \mu \mathrm{l}), \mathrm{ddH}_{2} \mathrm{O}(9.5 \mu \mathrm{l})$ and cDNA $(2 \mu \mathrm{l})$. PCR was performed under the following conditions: $95^{\circ} \mathrm{C}$ for $10 \mathrm{~min} ; 30$ cycles of $95^{\circ} \mathrm{C}$ for $30 \mathrm{sec}, 60^{\circ} \mathrm{C}$ for $30 \mathrm{sec}$ and $70^{\circ} \mathrm{C}$ for $30 \mathrm{sec}$. Conventional PCR was performed with $4 \mu \mathrm{l} \mathrm{cDNA}$, $12.5 \mu \mathrm{l}$ Taq MasterMix (CWBIO, Beijing, China), $1 \mu \mathrm{l}$ of each forward and reverse primer $(10 \mu \mathrm{M})$, and RNase-free water to a final $25 \mu \mathrm{l}$. Conventional PCR amplification was performed with a PTC-200 Peltier Thermal Cycler (MJ Research; Bio-Rad Laboratories, Inc., Hercules, CA, USA). The following primers were used: MSK1 forward, 5'-CCTCAAGACCCCATGCTT CA-3' and reverse, 5'-ACTTCTGTCATGGGACTGGA-3'; and GAPDH forward, 5'-GAGGCCGGTGCTGAGTATGT-3' and reverse, 5'-GGTGGCAGTGATGGCATGGA-3'. GAPDH was

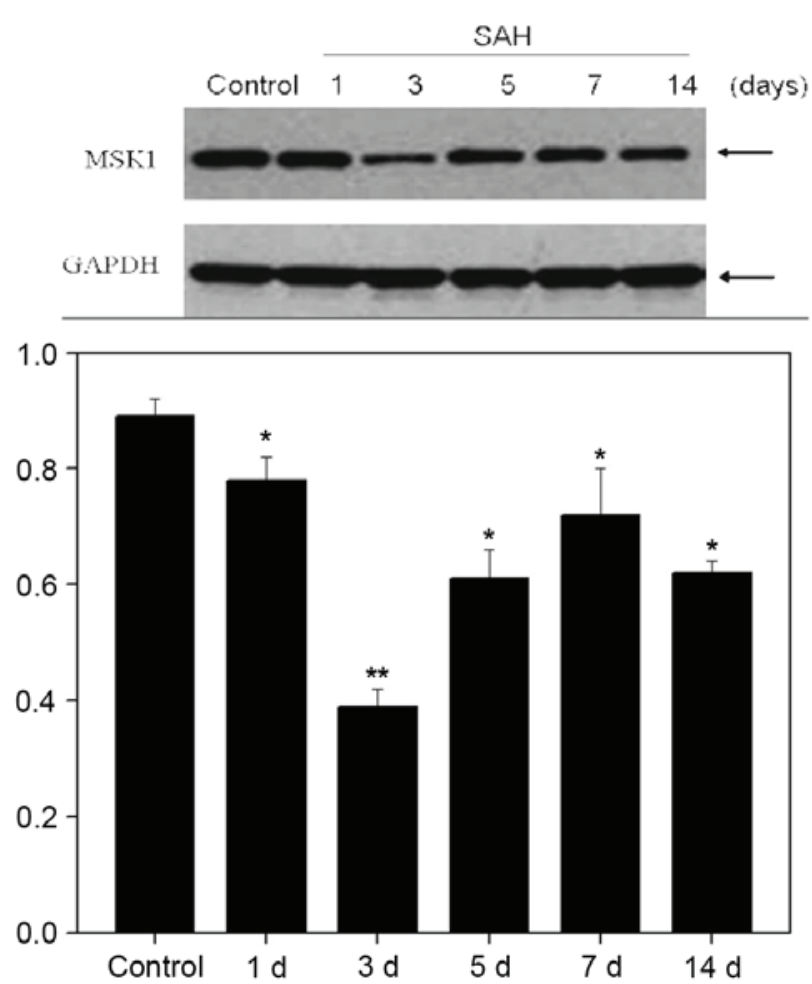

Figure 2. Western blot analysis of MSK1 in brains at various times subsequent to SAH. The expression of MSK1 in the cortex gradually reduced to a low at 3 days and recovered over the following days. MSK1, mitogen- and stress-activated protein kinase 1; SAH, subarachnoid hemorrhage. Values are presented as the mean \pm standard deviation of triplicate data. ${ }^{*} \mathrm{P}<0.05$ and ${ }^{* * *} \mathrm{P}<0.01$ vs. the control group.

used as an endogenous control and the $2^{-\Delta \Delta C q}$ method was used to quantify relative mRNA expression (20).

Statistical analysis. At least three replicates were performed per condition in each experiment. All values are expressed as the mean \pm standard error of the mean. SPSS version 21.0 (IBM SPSS, Armonk, NY, USA) was used for statistical analysis of the data. The statistical significance of differences between groups was determined by the Kruskal-Wallis test and Dunnett's multiple comparison test. $\mathrm{P}<0.05$ was considered to indicate a statistically significant difference.

\section{Results}

Expression of MSK1 is reduced at the protein and mRNA levels following SAH. MSK1 exhibits a high-level expression in the nervous system (18), however the function has not been well understood. Western blot analysis, immunohistochemistry, and conventional and quantitative PCR were performed in order to investigate the expression profiles of MSK1 in the cortex of rats following simulated $\mathrm{SAH}$.

Western blot analysis at 1, 3, 5, 7 and 14 days demonstrated that the expression of MSK1 gradually reduced to a low point at 3 days after $\mathrm{SAH}$, and recovered on day 5 onwards $(\mathrm{P}<0.05$; Fig. 2). Immunohistochemical staining on cross sections of the rat brain also demonstrated the differential expression of MSK1 between the control and SAH groups at 3 days. Abundant 


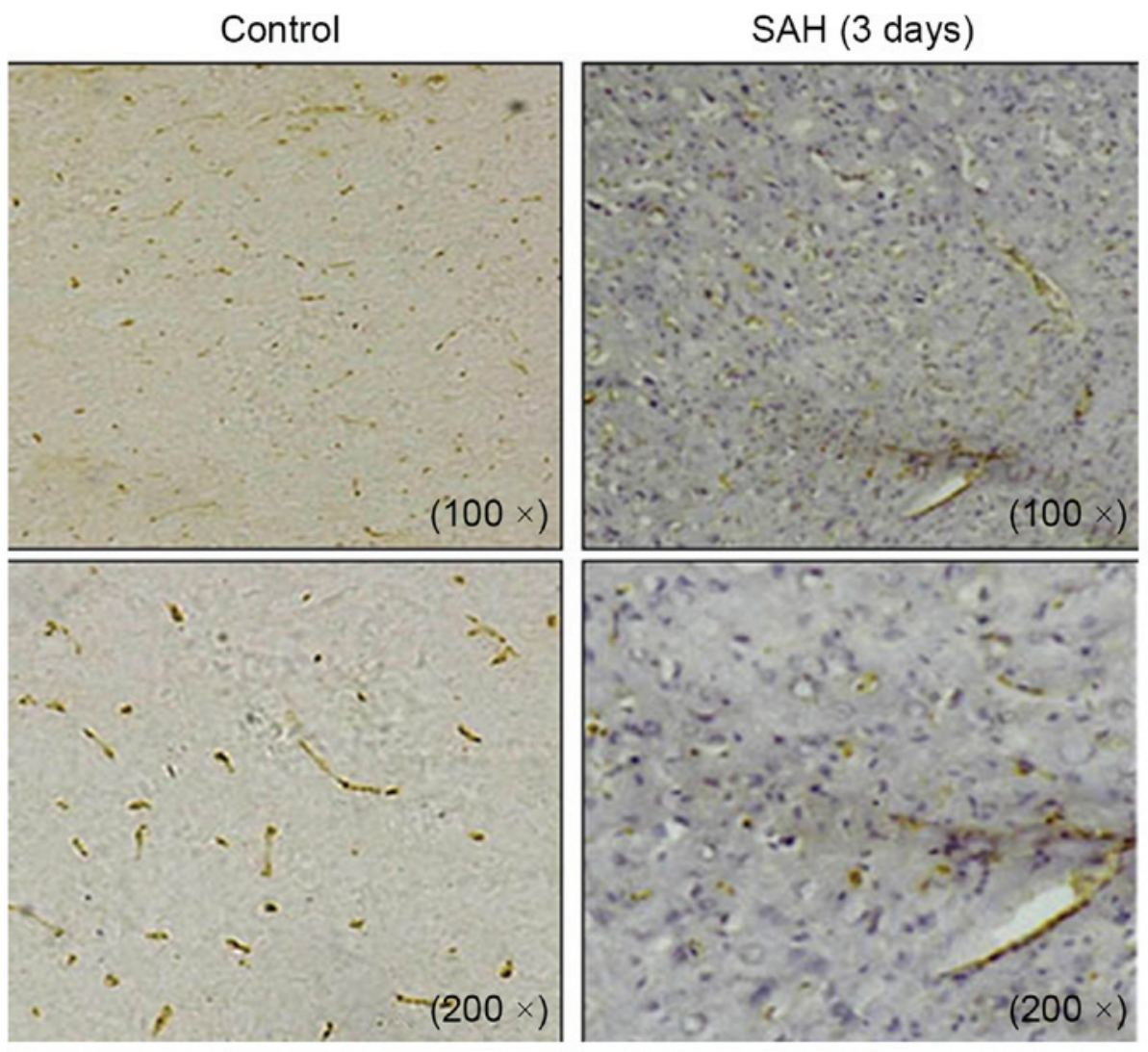

Figure 3. Immunohistochemical staining of MSK1 in cross-sections of rat brain. Low (x100) and high (x200) magnification views of cross-sections stained with MSK1 antibody in the control and 3 day SAH groups. Abundant MSK1-positive cells were detected in the control group and MSK1 was greatly reduced in the brain at 3 days following SAH. MSK1, mitogen- and stress-activated protein kinase 1; SAH, subarachnoid hemorrhage.

A
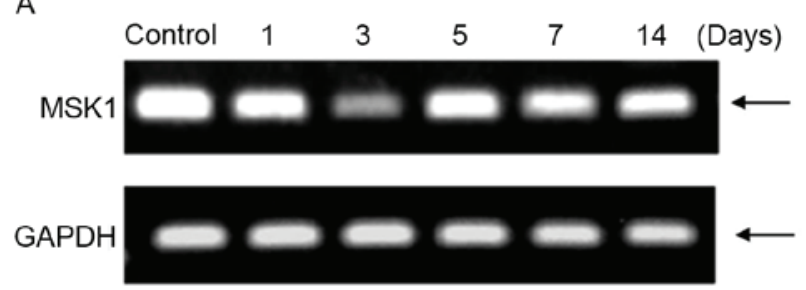

B

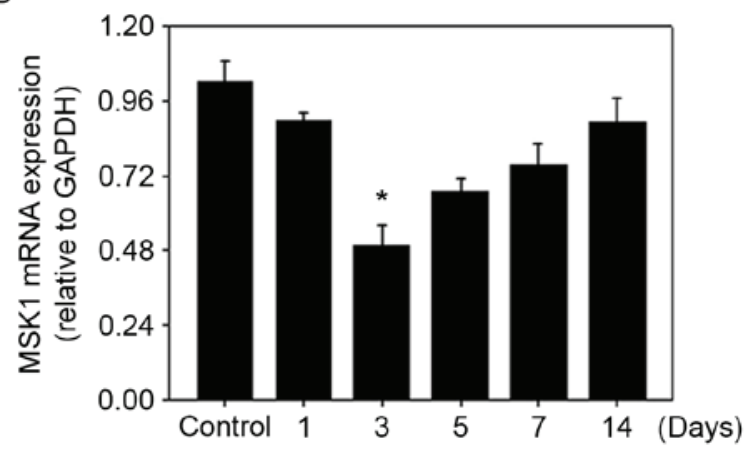

Figure 4. mRNA expression of MSK1 in rat cortexes at various times subsequent to SAH. (A) PCR analysis demonstrated the relatively high expression of MSK mRNA in the control group. The levels of MSK1 were reduced at all time points following SAH, reaching the minimum value at day 3. (B) There was no significant difference between the control, day 1 and day 14. Values are presented as the mean \pm standard deviation of triplicate data. ${ }^{*} \mathrm{P}<0.05$ and ${ }^{* *} \mathrm{P}<0.01$ vs. the control group.
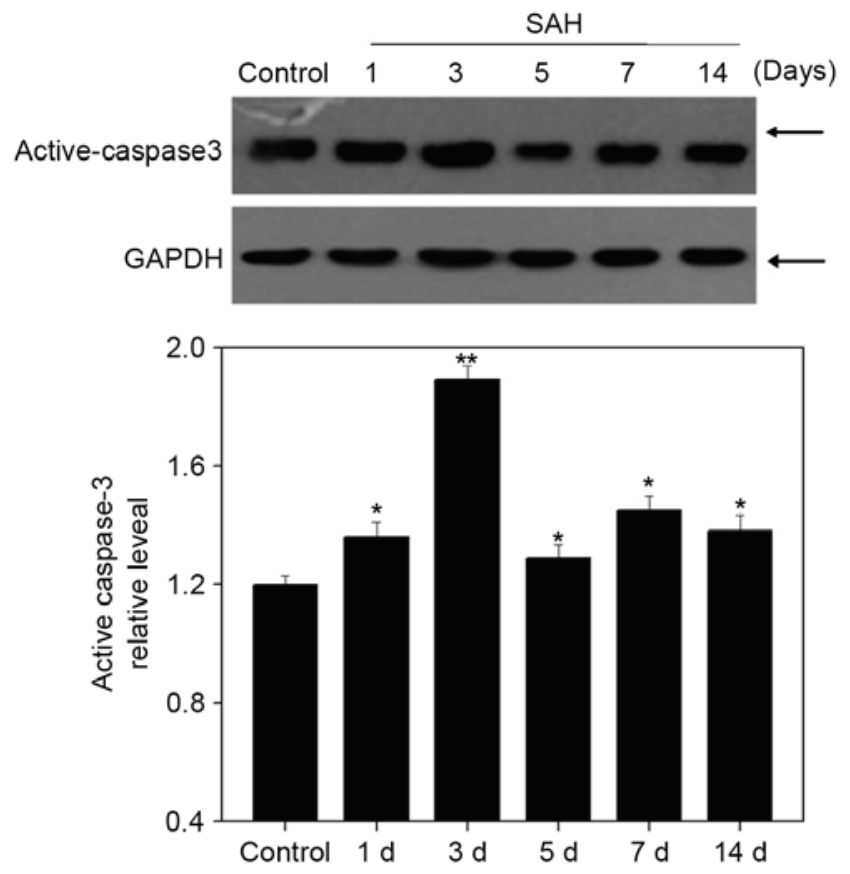

Figure 5. Western blot analysis of the protein expression of active caspase-3 in rat cortexes at various times subsequent to SAH. The expression of active caspase-3 was elevated subsequent to $\mathrm{SAH}$, reaching a peak at 3 days, and was reduced over the following days. SAH, subarachnoid hemorrhage. Values are presented as the mean \pm standard deviation of triplicate data. ${ }^{*} \mathrm{P}<0.05$ and ${ }^{* *} \mathrm{P}<0.01$ vs. the control group. 

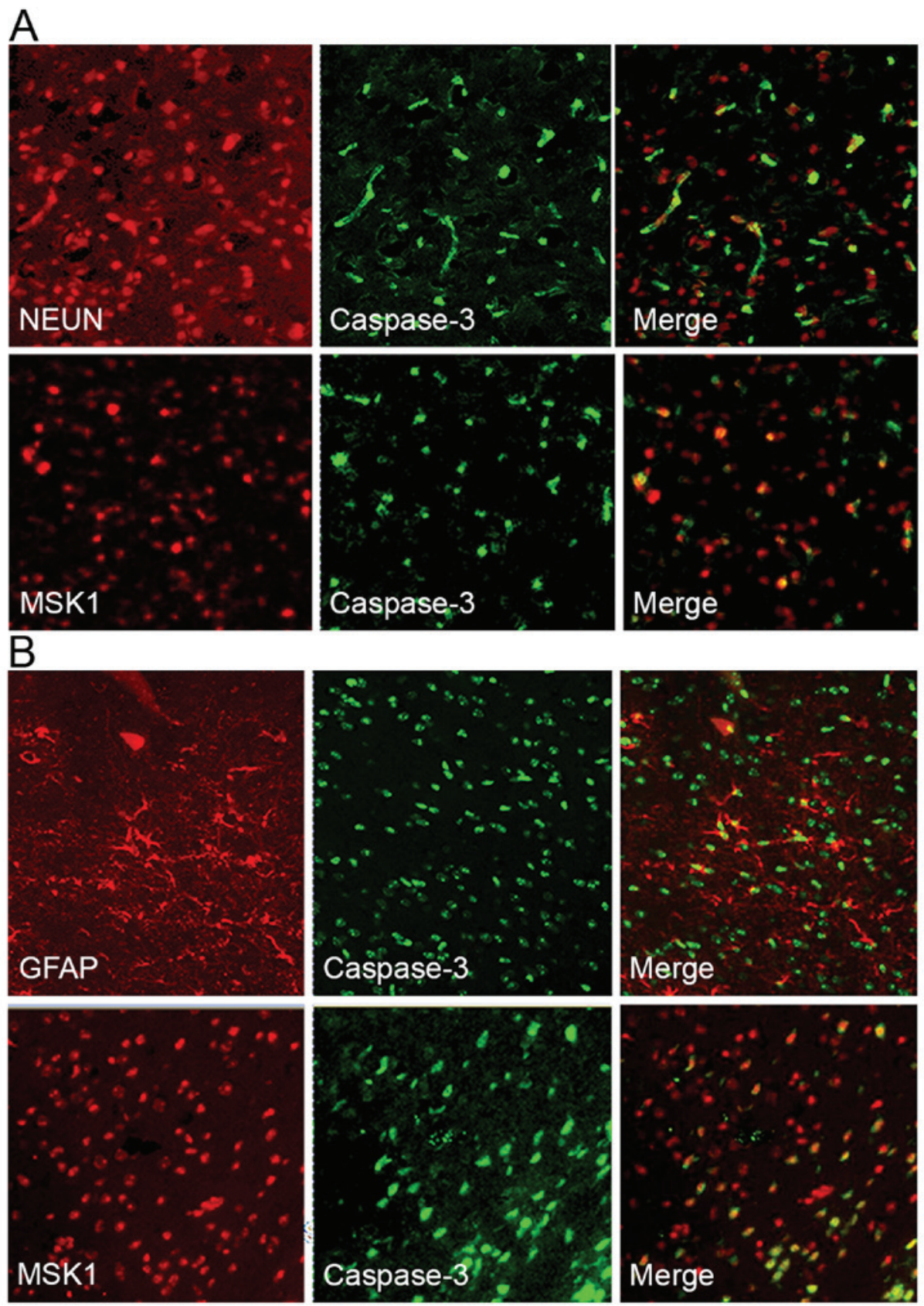

Figure 6. Double immunofluorescence staining. (A) Double immunofluorescence staining of MSK1, active caspase-3 and NeuN in the rat cortex subsequent to SAH. Active caspase-3 and NeuN were co-localized in the brain at 3 days after SAH, indicating that neurons were undergoing apoptosis. MSK1 fluorescence overlapped with active caspase-3 fluorescence. (B) Double immunofluorescence staining for active caspase-3, GFAP, and MSK1 in the brain cortex following SAH. The co-localization of active caspase-3 with GFAP was demonstrated. The majority of reactive astrocytes were active caspase-3 positive 3 days after $\mathrm{SAH}$, indicating that astrocytes were undergoing apoptosis. There was co-localization between active caspase-3 and MSK1. Scale bars represent $20 \mu \mathrm{m}$. MSK1, mitogen- and stress-activated protein kinase 1; NeuN, neuronal nuclear antigen; SAH, subarachnoid hemorrhage; GFAP, glial fibrillary acidic protein.

MSK1-positive cells were detected in the control group, whereas MSK1 positivity was visibly reduced in the brain at 3 days after SAH (Fig. 3). MSK1 mRNA was reverse transcribed and the relative level was assessed with $\mathrm{qPCR}$ and conventional PCR. MSK1 mRNA was expressed at a relatively high level in the control group and was significantly decreased at 3 days in the SAH group. The expression level reached the lowest point at 3 days after $\mathrm{SAH}$, and recovered over the following days, similar to the western blot result (Fig. 4). The results indicated that MSK1 may be associated with SAH-induced brain damage.
Detection of neuron and astrocyte apoptosis following SAH. Apoptosis serves a vital function in the control of cell numbers and removal of damaged cells. A critical step in the apoptosis process is the activation of caspases. The effect of MSK1 on the apoptosis of neurons and astrocytes in the CNS following SAH remains unclear.

A western blot analysis was performed to examine the expression of active caspase-3 (Fig. 5). Its expression was gradually elevated after $\mathrm{SAH}$ to a peak at 3 days $(\mathrm{P}<0.05)$, which was negatively associated with MSK1 expression (Fig. 2). 
In order to examine the association between MSK1 and apoptotic neurons at 3 days following SAH, double immunofluorescence staining for active caspase-3 and MSK1 or NeuN was performed. Active caspase-3 and NeuN were observed to co-localize, suggesting that neurons were undergoing apoptosis. Furthermore, when MSK1 and active caspase-3 fluorescence images were merged, MSK1 and active caspase-3 appeared to frequently co-localize (Fig. 6A).

To assess the association between MSK1 and apoptotic astrocytes, double immunofluorescence staining for GFAP or MSK1 and active caspase-3 was performed. GFAP and active caspase-3 immunoreactivity were observed in the cortex at day 3 post-SAH. Double labeling revealed the co-localization of active caspase- 3 with GFAP, suggesting that there were apoptotic astrocytes. Additionally, MSK1 expression was observed in a number of apoptotic astrocytes as evaluated by active caspase-3 staining. MSK1 reactivity coincided with astrocyte apoptosis in adjacent serial sections (Fig. 6B). These data indicated that MSK1 may serve a role in the apoposis of neurons and astrocytes following SAH.

\section{Discussion}

SAH is accompanied by a number of complicated molecular mechanisms in the brain which may result in ongoing cellular damage, the formation of scar tissue and neurological dysfunction. Several events, including increased intracranial pressure, transient global ischemia and blood clots obstructing the cerebral vasculature, may be responsible for the development of brain damage following SAH $(21,22)$. Experimental evidence supports that vasospasms induce neurological deterioration in the processes of secondary brain dysfunction $(23,24)$. However, clinical evidence suggests that certain patients deteriorate neurologically without vasospasms subsequent to $\mathrm{SAH}$ and preventing vasospasms does not always improve the patient outcome (9). A possible reason is that other processes injure neurons following SAH. Previous reseach has revealed that the delayed apoposis of neurons and astrocytes may be an important reason for a poor outcome in patients with SAH $(10,11)$. The purpose of the present study was to identify if MSK1, a protein from a particularly interesting family of MAPKs, is associated with this process, and to outline future possibilities of for improving the treatment of patients subsequent to SAH.

MSK1 contains two kinase domains and can be activated in vivo downstream of the MAPK $2 /$ mitogen-activated protein kinase 1 or MAPK11/14 cascades by the phosphorylation of Thr581, located within the C-terminal kinase domain (15). Once activated, the N-terminal domain phosphorylates substrates including $\mathrm{NF} \kappa \mathrm{B}, \mathrm{CREB}$, histone subunit $\mathrm{H} 3$, and HMGN1. In the present study, the dynamic changes to MSK1 expression following SAH were investigated with a model of autologous blood injections. Double immunofluorescence staining revealed that MSK1 expression occurred in neurons and astrocytes at 3 days following SAH. These data correspond with the hypothesis that MSK1 is associated with the pathophysiology of the CNS following SAH. Furthermore, it can be concluded that MSK1 might perform an important role the molecular mechanisms of brain damage subsequent to SAH.

MSKs regulate the IE genes, plasticate neuronal synapses and accommodate cytokine production. It was previously established that MSK1 represents a novel type of anti-cell death gene, which may enhance the phosphorylation of Bcl2-associated agonist of cell death (Bad) (25). The phosphorylation of Bad at Ser112 in response to growth factors or cytokines is a common mechanism for cell survival. The knockdown of MSK1 suppressed Bad phosphorylation subsequent to calcium ionophore A23187 treatment in neuronal cells in a previous study (18). In the present study, the expression of active caspase-3, which can initiate and effect the process of apoptosis, was negatively correlated with MSK1. Double immunofluorescence staining demonstrated that active caspase- 3 and $\mathrm{NeuN}$ were co-localized in the rat brain at 3 days subsequent to SAH. Furthermore, MSK1 fluorescence overlapped with active caspase-3 fluorescence. These data suggested that MSK1 may be associated with neuronal apoptosis subsequent to SAH. The detailed mechanisms for this require further study.

Astrocytes are one of the main types of cell that constitute the normal CNS parenchyma. CNS regeneration requires a largely astrocytic environment (26). However, the role of the astrocyte is under debate. Although astrocytes secrete important growth factors for neurons and prevent damage signals from spreading throughout the brain, the role following CNS injury appears detrimental to neuronal survival, axonal outgrowth and remyelination, preventing repair processes (27-29). The data of the present study revealed the co-localization of MSK1/active caspase-3 and GFAP/active caspase-3 in the brains at 3 days subsequent to SAH. These data indicated that MSK1 may serve a function in the procedure of astrocytic apoposis subsequent to SAH. Though the same comments as the previous paragraph regarding neuronal death apply, the cause of astrocyte apoposis following SAH remains unknown.

In summary, the present study revealed, for the first time, the expression of MSK1 following SAH. The co-localization and correlating changes in expression of MSK1/active caspase-3 at neurons and astrocytes indicated that MSK1 downregulation may contribute to SAH-induced apoptosis. To further understand the effect of MSK1 in the diversity of responses that may occur subsequent to $\mathrm{SAH}$ is a challenge for future investigations.

\section{References}

1. Cahill J, Calvert JW and Zhang JH: Mechanisms of early brain injury after subarachnoid hemorrhage. J Cereb Blood Flow Metab 26: 1341-1353, 2006.

2. Schuette AJ and Barrow DL: Epidemiology and long-term mortality in subarachnoid hemorrhage. World Neurosur 80: 264-265, 2013.

3. Ingall TJ and Whisnant JP: Epidemiology of Subarachnoid Hemorrhage. In: Yanagihara T, Piepgras DG and Atkinson JLD (eds): Subarachnoid Hemorrhage. Medical and Surgical Management. Marcel Dekker, New York, 63-78, 1998.

4. Taylor TN, Davis PH, Torner JC, Holmes J, Meyer JW and Jacobson MF: Lifetime cost of stroke in the United States. Stroke 27: 1459-1466, 1996.

5. Epidemiology of aneurysmal subarachnoid hemorrhage in Australia and New Zealand: Incidence and case fatality from the australasian cooperative research on subarachnoid hemorrhage study (ACROSS). Stroke 31: 1843-1850, 2000.

6. van Gijn J, Kerr RS and Rinkel GJ: Subarachnoid haemorrhage. Lancet 369: 306-318, 2007.

7. Kooijman E, Nijboer $\mathrm{CH}$, van Velthoven CT, Mol W, Dijkhuizen RM, Kesecioglu J and Heijnen CJ: Long-term functional consequences and ongoing cerebral inflammation after subarachnoid hemorrhage in the rat. PLoS One 9: e90584, 2014. 
8. Nolan CP and Macdonald RL: Can angiographic vasospasm be used as a surrogate marker in evaluating therapeutic interventions for cerebral vasospasm? Neurosurg Focus 21: E1, 2006.

9. Macdonald RL, Kakarieka A, Mayer SA, Pasqualin A Rufenacht DA, Schmiedek P and Kassell NF: Prevention of cerebral vasospasm after aneurysmal subarachnoid hemorrhage with clazosentan, an endothelin receptor antagonist. Neurosurgery 59: $453,2006$.

10. Hansen-Schwartz J, Vajkoczy P, Macdonald RL, Pluta RM and Zhang JH: Cerebral vasospasm: Looking beyond vasoconstriction. Trends Pharmacol Sci 28: 252-256, 2007.

11. Macdonald RL, Pluta RM and Zhang JH: Cerebral vasospasm after subarachnoid hemorrhage: The emerging revolution. Nat Clin Pract Neurol 3: 256-263, 2007.

12. Deak M, Clifton AD, Lucocq LM and Alessi DR: Mitogen- and stress-activated protein kinase-1 (MSK1) is directly activated by MAPK and SAPK2/p38, and maymediate activation of CREB EMBO J 17: 4426-4441, 1998.

13. Pierrat B, Correia JS, Mary JL, Tomás-Zuber M and Lesslauer W: RSK-B, a novel ribosomal S6 kinasefamily member, is a CREB kinase under dominant control of p38amitogen-activated protein kinase (p38aMAPK). J Biol Chem 273: 29661-29671, 1998.

14. Drobic B, Espino PS and Davie JR: Mitogen- and stress-activated protein kinase lactivity and histone $\mathrm{h} 3$ phosphorylation in oncogene-transformed mouse fibroblasts. Cancer Res 64: 9076-9079, 2004.

15. Arthur JS: MSK activation and physiological roles. Front Biosci 13: 5866-5879, 2008.

16. Johansen KM and Johansen J: Regulation of chromatin structure by histone H3S10 phosphorylation. Chromosome Res 14 393-404, 2006.

17. Deak M, Clifton AD, Lucocq LM and Alessi DR: Mitogen- and stress-activated protein kinase-1 (MSK1) is directly activated by MAPK and SAPK2/p38, and may mediate activation of CREB EMBO J 17: 4426-4441, 1998.

18. Clark CJ, McDade DM, O'Shaughnessy CT and Morris BJ: Contrasting roles of neuronal Msk1 and Rsk2 in Bad phosphorylation and feedback regulation of Erk signalling. J Neurochem 102: 1024-1034, 2007.
19. Ning B, Li Z, Zhu N, Hou G and Pang Q: Traumatic brain injury induces a downregulation of MSK1 in rat brain cortex. J Mol Neurosci 49: 380-386, 2013

20. Livak KJ and Schmittgen TD: Analysis of relative gene expression data using real-time quantitative PCR and the 2(-Delta Delta C(T) ) method. Methods 25: 402-408, 2001

21. Prunell GF, Svendgaard NA, Alkass K and Mathiesen T: Delayed cell death related to acute cerebral blood flow changes following subarachnoid hemorrhage in the rat brain. J Neurosurg 102: 1046-1054, 2005.

22. Jeon H, Ai J, Sabri M, Tariq A and Macdonald RL: Learning deficits after experimental subarachnoid hemorrhage in rats. Neuroscience 169: 1805-1814, 2010.

23. Dziurdzik P, Krawczyk L, Jalowiecki P, Kondera-Anasz Z and Menon L: Serum interleukin-10 in ICU patients with severe acute central nervous system injuries. Inflamm Res 53: 338-334, 2004.

24. Ostrowski RP, Colohan AR and Zhang JH: Molecular mechanisms of early brain injury after subarachnoid hemorrhage. Neurol Res 28: 399-414, 2006.

25. She QB, Ma WY, Zhong S and Dong Z: Activation of JNK1, RSK2, and MSK1 is involved in serine 112 phosphorylation of Bad by ultraviolet B radiation. J Biol Chem 277: 24039-24048, 2002.

26. Fawcett JW and Asher RA: The glial scar and central nervoussystem repair. Brain Res Bull 49: 377-391, 1999.

27. Franklin RJM and Ffrench-Constant C: Remyelination in the CNS: From biology to therapy. Nat Rev Neurosci 9: 839-855, 2008.

28. Segovia KN, McClure M, Moravec M, Luo NL, Wan Y, Gong X, Riddle A, Craig A, Struve J, Sherman LS and Back SA: Arrested oligodendrocyte lineage maturation in chronic perinatal white matter injury. Ann Neurol 63: 520-530, 2008.

29. Fawcett JW: Astrocytic and neuronal factors affecting axon regeneration in the damaged central nervous system. Cell Tissue Res 290: 371-377, 1997. 